\title{
Case Report: Fascial Treatment in Frozen Shoulder: A Case Report
}

\author{
Arsalan Ghorbanpour ${ }^{1 *}$ (1) \\ 1. Department of Physiotherapy, School of Rehabilitation, Tehran University of Medical Sciences, Tehran, Iran.
}

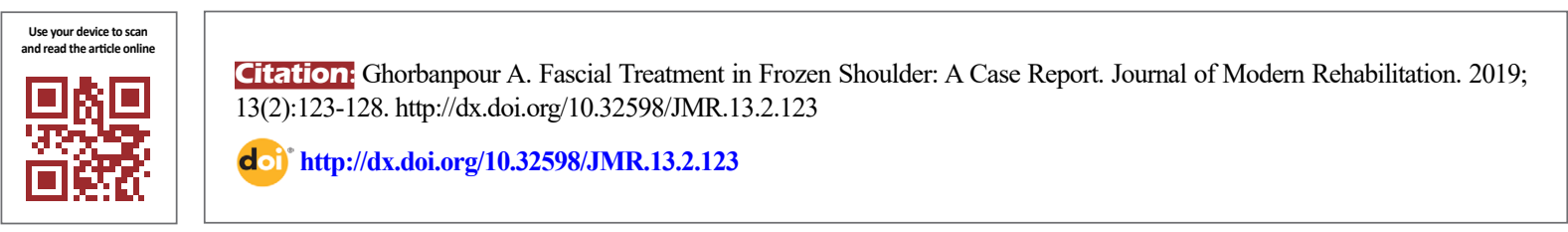

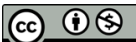

Article info:

Received: 03 Oct 2018

Accepted: 14 Feb 2019

Available Online: 01 Apr 2019

\section{Keywords:}

Fascia, Fascia training, Fascial distortion models, Fascia manipulation

\section{ABSTRACT}

Introduction: Frozen shoulder or adhesive capsulitis disorder is a progressive painful shoulder condition associated with stiffness and functional restriction of both active and passive shoulder motion, especially in abduction and external rotation.

Case Description: A 38-year-old man presented with right frozen shoulder started 3 months before admission. The patient reported no change in his symptoms after the previous 10 sessions of conventional physiotherapy. Physical examination findings were significant for both active and passive range of motion with 8 out of 10 in pain score (Visual Analog Scale (VAS); 8/10) For the treatment, we applied the fascial treatment, including the Fascial Distortion Model (FDM) and fascial training. After 10 sessions, the shoulder range of motion restored to $140^{\circ}$ abduction and $90^{\circ}$ external and internal rotation with no pain (VAS; 0/10).

\section{Introduction}

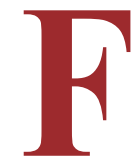

rozen shoulder, clinically known as adhesive capsulitis disorder, is a progressive painful shoulder condition associated with stiffness and functional restriction of both active and passive shoulder motion [1-3]. The condition causes a marked reduction in flexion and external rotation of the shoulder joint with the disturbance of sleep on the affected side and escalating pain at night [1]. In pathological studies, frozen shoulder is associated with the chronic inflammation of the synovial layer of the joint and capsule thickening, fibrosis, and contracture $[1,3]$. The prevalence of the frozen shoulder is about $2 \%$ to $5 \%$ of the general population, mainly seen in the middle age group [3]. According to Codman, the symptoms of the frozen shoulder are "slow and spontaneous onset, pain near the insertion of deltoid, inability to sleep on the affected side, painful and incomplete shoulder elevation and external rotation, and loss of the normal axillary recess" $[1,3,4]$.

Based on the recent anatomical and histological studies, fascial dysfunction is one of the causes of frozen shoulder. The painful contractures, especially around periarticular connective tissues, are mostly associated with increased stiffness in fascial tissue in joints. Accumulation of fibroblasts or contractile myofibroblasts

* Corresponding Author:

Arsalan Ghorbanpour, PhD Candidate.

Address: Department of Physiotherapy, School of Rehabilitation, Tehran University of Medical Sciences, Tehran, Iran.

Tel: +98 (911) 2936144

E-mail: ghorbanpoura@razi.tums.ac.ir, arsalanghorbanpour@gmail.com 
and periarticular fascial layers has been reported at the past or present inflammatory processes around the frozen shoulder [5-9]. In this disorder, a dense matrix of type III collagen populated with fibroblasts and myofibroblasts is seen in the capsule, which thickens the shoulder joint capsule and coracohumeral ligament within the rotator interval $[5,6,8]$. Also, a few studies reported increasing vascularity, fibrosis, hyalinization, vascular villous synovitis, and mature scar tissue in the periarticular connective tissues [5].

Management and treatment of frozen shoulder are mainly non-operative, including physiotherapy and drug therapy (intra-articular steroid injections and nonsteroidal anti-inflammatory drugs). Physiotherapy consists of modalities (Transcutaneous Electrical Nerve Stimulator (TENS), cold or warm pads, and so on), therapeutic exercises (stretching and strength program), and manual therapy (mobilization and manipulation techniques, muscle energy techniques, fascial release techniques and so on) [10-12].

Different fascial release techniques, as hands-on techniques, exist in the world. Two essential fascial release techniques are the fascial training and the Fascial Distortion Model (FDM) [13-16]. The fascial training consists of techniques for relaxing myofibroblasts and alphasmooth muscle actin cells and re-aligning fascia layers with the unloading of fascia $[13,14]$. FDM consists of an analogy of assessment and treatment of fascial dysfunctions. FDM identifies six different distortions or alterations of fascia and fascial layers: Trigger-band (TB), folding distortion, Herniated Trigger-point (HTP), continuum distortion, cylinder distortion, and Tectonic Fixation (TF). Therapists differentiate these six distortions by asking their patients who describe and indicate the nature and location of pain $[16,17]$. Both techniques are

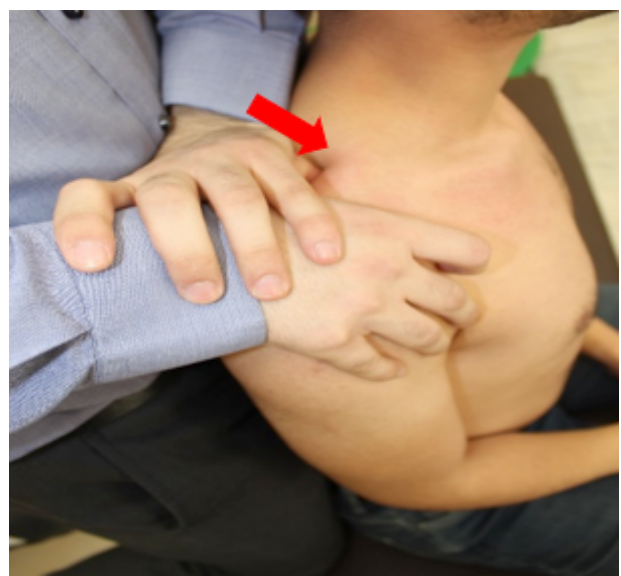

JMR

Figure 1. Fascial distortion model for herniated Trigger-point used for relieving pain and restoring joint movements and muscle coordination $[14,16]$.

The purpose of this case report is to describe fascia realignment with using the fascial training and the fascial distortion model as a conservative intervention in the frozen shoulder.

\section{Case Description}

A 38-year-old man (height: $171 \mathrm{~cm}$, weight: $91 \mathrm{~kg}$ ) referred to our clinic in April 2019. His chief complaint was right shoulder pain and restriction of movement since 3 months ago. According to him, the symptoms started after abdominal operation 9 months ago. His pain got worse at night and disrupted his sleep. The patient reported difficulty working as a typist, writing, playing guitar, and putting on his clothes over his head. The patient tended to place his arm near the body. His pain was measured based on the Visual Analog Scale (VAS) which was 8 out of 10 in daily activities. The patient also reported that his symptoms and pain became slightly better after 10 sessions of conventional treatment, including infrared radiation, ultrasound, conventional TENS, rotator cuff muscles stretching, and strengthening exercises. However, he reported self-treatment using non-steroidal anti-inflammatory and analgesic drugs for the last 2 months.

The patient described the location and nature of his pain by drawing a line from the trapezius region and anterior glenohumeral joint to the anterior of the middle arm and reported the nature of pain as a burning or pulling muscles. In addition, he pushed his index finger of one hand into a painful point over the middle of anterior and middle deltoid and supraspinatus and upper trapezius muscles. The patient felt deep pain, numbness and tingling in the glenohumeral

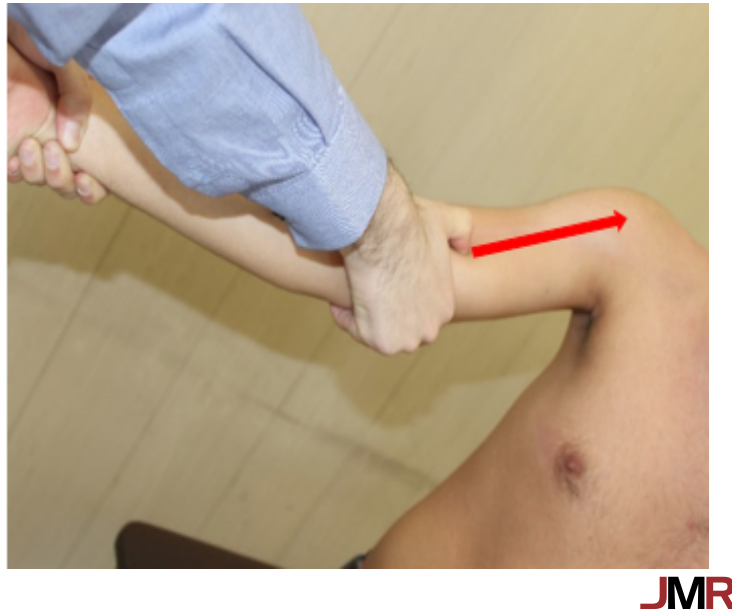

Figure 2. Fascial distortion model for Trigger-band distortion 


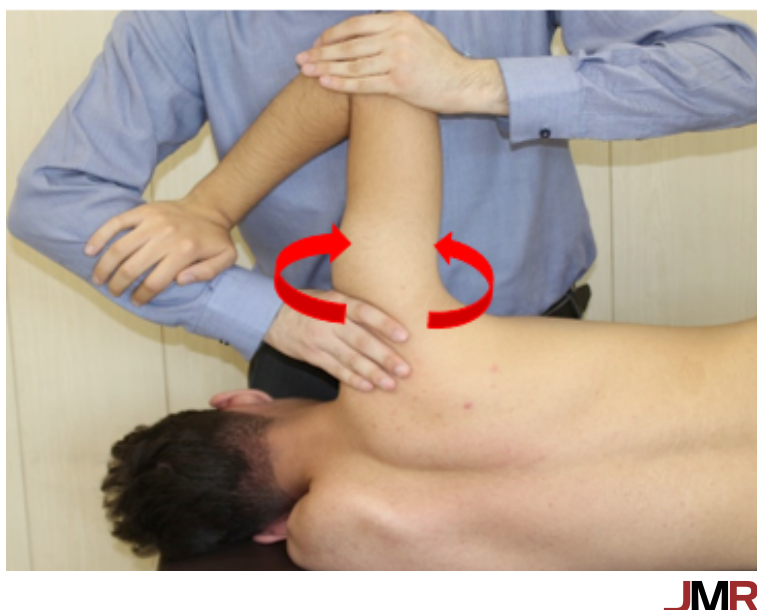

Figure 3. Fascial release for improving joint lubrication and ability the fascial gliding

joint, and he tended to catch his right shoulder with the palm of the left hand for squeezing this area to relieve pain slightly. The patient had no remarkable medical history or underlying diseases.

Physical examination findings were significant for both active and passive range of motion and atrophy of the right supraspinatus and deltoid muscles. The patient reported increased pain with passive shoulder elevation and external rotation. In addition, his active and passive range of motion (measured by a goniometer) decreased to $90^{\circ}$ forward flexion and abduction, $70^{\circ}$ internal rotation in abduction, and only a little external rotation in abduction. Aside from these restrictions, his other shoulder and elbow movements were normal. The range of motion and quality of neck movement was slightly reduced in left rotation $\left(75^{\circ}\right)$ and side bending $\left(30^{\circ}\right)$. The patient reported feeling pain and tight muscle on the upper trapezius region at the end of the neck left rotation and side bending range of motion. The patient reported feeling tenderness during palpation of deltoid attachment, glenohumeral, and acromioclavicular joint. Also, his pectoralis minor and upper trapezius muscles were spastic. All muscles in the shoulder girdle were strong and painless, except supraspinatus, subscapularis, and anterior deltoid muscles (4 out of 5). All reflexes and sensations were normal.

Special tests for assessing neck disorders revealed normal results. The empty can test result was positive on the right shoulder. No evidence of shoulder joint instability or impingement was found. He had tender points on the upper trapezius, supraspinatus, and pectoralis minor at the right side. Magnetic resonance imaging showed a slight thickening in the joint capsule and the coracohumeral ligament and mild rotator cuff tearing, but spe- cial tests for diagnosis of rotator cuff tear were negative No evidence of somatic dysfunction was found in the thoracic, lumbar spine, or sacroiliac joint. Based on the symptoms and physical examination findings, his condition was diagnosed as frozen shoulder.

The routinely therapeutic intervention in the first sessions included TENS modalities for pain reduction (with a pulse frequency of $120 \mathrm{~Hz}$, pulse duration of $80 \mu$ s for 15 minutes) and supraspinatus (with a pulse frequency of $100 \mathrm{~Hz}$, pulse duration of $150 \mu$ s for 12 minutes), hot packs. The therapeutic exercises included the range of motion exercises with wand and pulley, scapula muscles re-balancing exercises (shoulder shrugging in standing and supine), hip abduction, and extension without and with thera band, and the diagonal patterns in proprioceptive neuromuscular facilitation exercise without and with thera band. In the first session, the grade I and II oscillation mobilizations were used for pain reduction and determination of sensitivity to manual therapy. Strain/Counterstrain technique was used on the tender points on the upper trapezius, supraspinatus, and pectoralis minor. After the first three sessions, mobilization with movement techniques was applied for five sessions to improve the range of motion in the 4-point kneeling position. The therapist with one hand, moved scapula to depression, retraction, and downward rotation and maintained this position and approximated scapula and clavicle between the two hands. Then, the patient was asked to lean and sit backward on his heels.

FDM is applied based on the patient's body language to find the location of the pain. The patient pushed his index finger into the middle of the anterior and middle deltoid and supraspinatus and upper trapezius muscles. This body language means Herniated Trigger-point (HTP). With palpation, the therapist would find an irregularity and protrusion in these tissues. The therapist pressed the tip of his right thumb on the target tissue, exerted a direct pressure, and maintained it until the tissue went back through the fascial plane (Figure 1).

The patient also drew a line from the trapezius region and anterior glenohumeral joint to the anterior of the middle arm and reported the nature of pain as a burning or pulling muscles. This body language means Triggerband (TB). The therapist then applied firm pressure with the tip of his thumb, starting at the anterior of the middle arm and followed by painfully drawn a line to the trapezius region (Figure 2). The therapist continued this pressure in the opposite direction to realign the fascial fibers. In this technique, the patient initially felt pain with the 
therapist's thumb pressure but pain reduced and range of motion improved after applying these techniques.

In the frozen shoulder, FDM has a distortion called TF. For the treatment of TF, special manipulation is used for restoring the ability of fascial gliding and increasing synovial lubrication. However, because of his previous bad experience, the patient was afraid of any manipulation in the shoulder and did not allow the therapist to manipulate this area. Therefore, the therapist used another fascia release technique to restore the ability of fascial gliding.

The therapist applied fascial training for improving the ability of fascial gliding and lubrication in the glenohumeral joint. The patient was positioned in the lateral recumbent position or side-lying on the left shoulder. One hand of the therapist was placed over the acromioclavicular joint to stabilize the patient's shoulder. The other hand of the therapist was placed on the elbow to control the humerus and apply the fascial release technique. The technique was applied at the end of abduction and external rotation in the patients with the frozen shoulder (Figure 3 ). Then, the therapist slightly moved humerus clockwise or counterclockwise for 90 seconds until the shoulder range of motion increased. This technique was applied for 3-5 repetitions in each session. Both fascial treatments (FDM and fascial training) were used after the first three sessions.

On re-assessment, the patient's shoulder range of motion improved to $140^{\circ}$ abduction and $90^{\circ}$ external and internal rotation. Also, his pain decreased to 0 out of 10 in daily activities after 10 sessions of treatment. The therapist asked the patient to return to the physiotherapy clinic for follow-up in 3 weeks. At the follow-up session, the patient reported his satisfaction with his improvement and maintained his range of motion and pain, similar to the last treatment session. Besides, the shoulder examination revealed no significant findings.

\section{Discussion}

The results of the case report show that fascia treatment, FDM, and fascial training positively influence fascial dysfunction, decrease pain, and improve shoulder range of motion in the patient with frozen shoulder. Although pain reduction may be due to the combination of the other modalities or exercises, these treatments are used when the previous conventional physiotherapies have not achieved any improvement. The focus of this case report was to describe the effects of the combination of the FDM and fascial training in improving the frozen shoulder.
According to previous studies, one of the causes of frozen shoulder is the accumulation of fibroblasts or contractile myofibroblasts and periarticular fascial layers during the past or present inflammatory processes $[5,6]$. The fascial treatment or release helps improve fibroblast proliferation, normal collagen alignment, and normal gliding fascial layers $[11,13]$. The fascia is manipulated with direct or indirect methods, allowing the components of the fascia layers to reorganize themselves into a more flexible, functional fashion [18]. The fascial release or fascial training is one of the essential techniques in the dysfunction or mal-alignment of the deep layers of fascia. In the fascial training, the fascia is unloaded to relax, improve, soften, and lengthen the fascia through enhancing synovial lubrication [19]. Also, the FDM has a conservative and immediate effect of managing musculoskeletal disorders such as the frozen shoulder. However, there is no evidence about the long-term impact of the FDM, so it calls for more investigation.

According to this report, both fascia treatments can improve pain and range of motion of the patient's frozen shoulder. However, it is better to use a standard conservative intervention package such as myofascial release, mobilization, or therapeutic exercises.

\section{Ethical Considerations}

\section{Compliance with ethical guidelines}

All ethical principles were considered in this article.

Funding

This research did not receive any specific grant from funding agencies in the public, commercial, or not-forprofit sectors.

\section{Conflict of interest}

The author declared no conflict of interest.

\section{References}

[1] Dias R, Cutts S, Massoud S. Frozen shoulder. British Medical Journal. 2005; 331(7530):1453-6. [DOI:10.1136/ bmj.331.7530.1453] [PMID] [PMCID]

[2] Zuckerman JD, Rokito A. Frozen shoulder: A consensus definition. Journal of Shoulder and Elbow Surgery. 2011; 20(2):322-5. [DOI:10.1016/j.jse.2010.07.008] [PMID] 
[3] Kingston K, Curry EJ, Galvin JW, Li X. Shoulder adhesive capsulitis: Epidemiology and predictors of surgery. Journal of Shoulder and Elbow Surgery. 2018; 27(8):1437-43. [DOI:10.1016/j.jse.2018.04.004] [PMID]

[4] Lewis J. Frozen shoulder contracture syndrome - Aetiology, diagnosis and management. Manual Therapy. 2015; 20(1):2-9. [DOI:10.1016/j.math.2014.07.006] [PMID]

[5] Klingler W, Velders M, Hoppe K, Pedro M, Schleip R. Clinical relevance of fascial tissue and dysfunctions. Current Pain and Headache Reports. 2014; 18(8):439-45. [DOI:10.1007/ s11916-014-0439-y] [PMID]

[6] Stecco C, Macchi V, Porzionato A, Duparc F, De Caro R. The fascia: the forgotten structure. Italian Journal of Anatomy and Embryology. 2011; 116(3):127-38. [PMID]

[7] Kumka M, Bonar J. Fascia: A morphological description and classification system based on a literature review. The Journal of the Canadian Chiropractic Association. 2012; 56(3):179-91. [PMCID]

[8] Schleip R, Klingler W, Lehmann-Horn F. Active fascial contractility: Fascia may be able to contract in a smooth musclelike manner and thereby influence musculoskeletal dynamics. Medical Hypotheses. 2005; 65(2):273-7. [DOI:10.1016/j. mehy.2005.03.005] [PMID]

[9] Russell S, Jariwala A, Conlon R, Selfe J, Richards J, Walton M. A blinded, randomized, controlled trial assessing conservative management strategies for frozen shoulder. Journal of Shoulder and Elbow Surgery. 2014; 23(4):500-7. [DOI:10.1016/j.jse.2013.12.026] [PMID]

[10] Tasto JP, Elias DW. Adhesive capsulitis. Sports Medicine and Arthroscopy Review. 2007; 15(4):216-21. [DOI:10.1097/ JSA.0b013e3181595c22] [PMID]

[11] Page P, Labbe A. Adhesive capsulitis: Use the evidence to integrate your interventions. North American Journal of Sports Physical Therapy. 2010; 5(4):266-73. [PMID] [PMCID]

[12] Jason JI, Ganesh Sundaram S, Vengata Subramani M. Physiotherapy interventions for adhesive capsulitis of shoulder: A systematic review. International Journal of Physiotherapy and Research. 2015; 3(6):1318-25. [DOI:10.16965/ijpr.2015.198]

[13] Myers T. Fascial fitness: Training in the neuromyofascial web. IDEA Fitness Journal. 2011; 8(4):36-43.

[14] Schleip R, Müller DG. Training principles for fascial connective tissues: scientific foundation and suggested practical applications. Journal of Bodywork and Movement Therapies. 2013; 17(1):103-15. [DOI:10.1016/j.jbmt.2012.06.007] [PMID]

[15] Shah S, Bhalara A. Myofascial release. International Journal of Health Sciences and Research. 2012; 2(2):69-77.

[16] Typaldos S. Introducing the fascial distortion model American Academy of Ophthalmology Journal. 1994; 14-36.

[17] Thalhamer C. A fundamental critique of the fascial distortion model and its application in clinical practice. Journal of Bodywork and Movement Therapies. 2018; 22(1):112-7. [DOI:10.1016/j.jbmt.2017.07.009] [PMID]

[18] Hains G. Chiropractic management of shoulder pain and dysfunction of myofascial origin using ischemic compression techniques. The Journal of the Canadian Chiropractic Association. 2002; 46(3):192-200. [PMCID]
[19] Godges JJ, Mattson-Bell M, Thorpe D, Shah D. The immediate effects of soft tissue mobilization with proprioceptive neuromuscular facilitation on glenohumeral external rotation and overhead reach. Journal of Orthopaedic \& Sports Physical Therapy. 2003; 33(12):713-8. [DOI:10.2519/ jospt.2003.33.12.713] [PMID] 
This Page Intentionally Left Blank 